\title{
Integração de imagens de satélite e dados de GPR em um estudo sobre o sistema costeiro de Barra do Rio/RN
}

lurianne Monik Medeiros Conti (DGEF/UFRN), André Giskard Aquino da Silva (DG/UFRN), Josibel Gomes de Oliveira Júnior (DGEF/UFRN)

Copyright 2016, SBGf - Sociedade Brasileira de Geofísica

Este texto foi preparado para a apresentação no VII Simpósio Brasileiro de Geofísica, Ouro Preto, 25 a 27 de outubro de 2016. Seu conteúdo foi revisado pelo Comitê Técnico do VII SimBGt, mas não necessariamente representa a opinião da SBGf ou de seus associados. É proibida a reprodução total ou parcial deste material para propósitos comerciais sem prévia autorização da SBGf.

\section{Resumo}

This paper presents an integrated study based on satellite images and ground penetrating radar (GPR) in a coastal area located in northeastern Brazil to identify stratigraphic records associate them to coastal evolution. Four satellite images (dated from Dec-2001, Apr-2005, 2010 and 2015) were used to map the decadal morphodynamic behavior of a spit to understand the short term coastal changes adjacent to the Ceará Mirim River estuary. The GPR data shows sedimentary structures dipping to NW (associated to spit development), the contact between aeolian/beach sediments and a paleo-channel. In addiction the GPR identified a conductive zones related to saline intrusion.

Introdução

A praia de Barra do Rio, localizada às margens do Rio Ceará Mirim, no município de Extremoz-Rio Grande do Norte (RN) (Figura 1) é caracterizada por tabuleiros costeiros, dunas e planícies de inundação (Lima, 2011). Adicionalmente, o entorno do rio Ceará Mirim, apresenta características bastante dinâmicas, típicas de áreas de formação recente, com ventos predominantes na direção SE-NO e, em menor grau, na direção L-O (COSERN, 2003). Similarmente ao que ocorre em diversas partes do litoral Brasileiro (Lima \& Vital, 2002) e do mundo (Vilas et al., 1999). No Rio Grande do Norte existem complexos processos costeiros que podem ser melhor entendidos por meio da caracterização morfodinâmica da linha de costa. O alto grau morfodinâmico dos spits (comumente encontrados no litoral do $\mathrm{RN}$ ) os torna de grande importância no entendimento da dinâmica sedimentar local. A formação dessas feições ocorre devido a uma interação conjunta da ação das ondas, correntes, ventos e variações da maré. Neste contexto, utilizaremos imagens de satélite e GPR para estudar o crescimento de um spit localizado em Barra do Rio, buscando estabelecer relações entre a sua arquitetura deposicional e padrões locais de vento e maré. Espera-se que os resultados alcançados possam contribuir para um melhor entendimento acerca da dinâmica costeira local, fornecendo subsídios para a criação de modelos evolutivos.

\section{Metodologia/ Problema Investigado}

Neste trabalho foram utilizados, de forma integrada, imagens de satélite e dados de GPR para estudar a dinâmica costeira na foz do estuário do rio Ceará Mirim (Extremoz/RN). Quatro imagens de satélite obtidas através do Google Earth, dos anos de 2001(Google, 2001), 2005 (Google, 2005), 2010 (Google, 2010) e 2015
(Google, 2015), foram usadas para mapear a evolução do spit. A imagem de 2001(Figura 2A) foi usada como base para o georreferenciamento das demais imagens. Isto garante uma maior precisão na medição do crescimento do spit entre 2001 e 2015. O programa ArcGIS (ESRI, 2012) foi usado tanto para o georreferenciamento quanto para o mapeamento do contorno do spit. No contorno do spit foi feito o contato terra-água, levando-se em consideração a meia distância entre partes secas e úmidas, para minimizar o erro inerente à altura da maré nas diferentes datas de aquisição das imagens. Por fim, foi feito um cálculo de cobertura areal do spit, e seus entornos, para uma melhor visualização da relação sedimentação/erosão no local (Figura 2C).

O levantamento de GPR foi conduzido por Conti et al. (2015) e fez uso de uma antena blindada de $200 \mathrm{MHz}$. Adicionalmente, o equipamento foi configurado para adquirir um traço a cada $0.1 \mathrm{~m}$, na direção SE-NO (Figura 1). O dado bruto foi reprocessado (Xavier Neto, 2006) e reinterpretado com a finalidade de obter boas imagens de estruturas sedimentares relacionadas à dinâmica costeira local, em particular, ao crescimento do spit. Para isso, empregou-se o programa REFLEX 6.1.0 (Sandmeier, 2000). Os dados altimétricos, necessários para a correção topográfica, foram levantados com um GPS RTK, em modo cinemático.

\section{Resultados}

A dinâmica sedimentar na área de estudo foi bastante intensa entre 2001 e 2015. Dois spits são observados, um na margem leste e outro na margem oeste, que apresentam sentidos de crescimento opostos (Figuras $2 \mathrm{~A}$ e 2B). A configuração morfológica da região em 2001 (Figura 2A) será considerada como ponto inicial, e utilizado nos comparativos gerais da relação deposiçãoerosão. Adicionalmente, a Figura $2 \mathrm{C}$ sintetiza todas as relações de sedimentação/erosão discutidas a seguir.

Linearmente, ocorreu um deslocamento para NO da extremidade do spit leste de aproximadamente 296,1 m entre 2010 e 2015, o que representa uma taxa de migração de aproximadamente 59,2 m/ano. Entretanto, observa-se que esta variação não ocorreu de forma linear. O processo de deposição no spit leste foi mais acentuado neste período, porém, entre 2001 e 2005 não houve migração do spit leste. Neste período, houve um afinamento do spit leste com a erosão da sua margem voltada para 0 rio (deslocamento máximo de aproximadamente $57 \mathrm{~m}$ ) e a progradação de sua linha de costa (progradação máxima de aproximadamente $32 \mathrm{~m}$ ). Além disso, ocorreu o secionamento do spit oeste, e concomitante erosão da linha de costa da margem oeste do estuário do rio Ceará Mirim, a qual causou um recuo da linha de costa de aproximadamente $120 \mathrm{~m}$. Isto 
ocasionou o alargamento da foz do estuário, passando de $104 \mathrm{~m}$ (2001) para $214 \mathrm{~m}$ (2005) (Figura 2C).

Entre 2005 e 2010, ocorreu um processo de sedimentação na margem oeste da foz do estuário, que resultou num avanço de aproximadamente $30 \mathrm{~m}$ da linha de costa neste local. Houve a unificação das pequenas ilhas formadas pelo seccionamento do spit oeste, formando um único spit (Figura 2). Em 2010, o spit leste foi completamente erodido e a linha de costa teve um recuo máximo de 58,2 m (próximo a foz do estuário) (Figura 2C). Neste período houve um estreitamento da foz do estuário que passou de $214 \mathrm{~m}$ (2005) para $168 \mathrm{~m}$ (2010).

Entre 2010 e 2015 ocorreu um acelerado processo de deposição na foz do estuário, que ocasionou a migração de $296,1 \mathrm{~m}$ do spit leste e progradação de sua linha de costa de 48,4 m. A progradação do spit leste causou o estreitamento da foz do estuário, passando de $168 \mathrm{~m}$ (2010) para $67,5 \mathrm{~m}$ (2015). Além disso, houve o retrabalhamento dos sedimentos que compunha o spit oeste (Figura $2 \mathrm{C}$ ).

Os resultados obtidos com o reprocessamento da seção de GPR bruta (Figura 3A) podem ser vistos por meio das Figuras 3B (dado processado) e $3 C$ (interpretação de 3B). Observe que todas as elevações topográficas são relativas ao nível do mar.

Segundo Conti et al. (2015), ambientes costeiros são compostos por sedimentos arenosos de elevada resistividade elétrica, ideais para levantamentos com GPR. No entanto, a eventual presença de solos condutivos impõe uma forte atenuação ao pulso eletromagnético, dificultando 0 imageamento de estruturas em subsuperfície. Por meio da Figura 3C observa-se, uma marca verde que delimita uma zona cega. A ausência de refletores nessa zona permitiu interpretá-la como sedimentos arenosos saturados com água salgada (Conti et al., 2015). Ao longo de toda a seção de GPR também são observadas estruturas sedimentares mergulhando para NO (Ver interior dos retângulos azul e vermelha, figura $3 \mathrm{C}$ ), fato que não pode ser comprovado dentro da área sob influência da água salgada (Marca verde, figura $3 \mathrm{C}$ ). Por outro lado, entre as posições $0 \mathrm{~m}$ e $20 \mathrm{~m}$ (Marca vermelha, figura 3C), percebe-se que estas mesmas estruturas ocorrem em profundidades de até $3 \mathrm{~m}$. Confrontando estas observações com as imagens de satélite da área de estudo (Figura 1), percebe-se que a direção de migração do spit também ocorre de SO para NO, o que nos levou a interpretar estes refletores como um registro pretérito do crescimento do spit. Além das estruturas sedimentares acima descritas, por meio da Figura 3C, podem ser vistas duas importantes superfícies limitantes: A primeira é caracterizada por um forte refletor subparalelo, que corre entre as posições $35 \mathrm{~m}$ e $51 \mathrm{~m}$ (marca vermelha pontilhada), interpretado como 0 contato entre sedimentos eólicos e, provavelmente, o topo do spit. A segunda superfície limitante consiste em um refletor lateralmente contínuo, com concavidade voltada para cima (Figura 3C, marca amarela). A julgar por sua geometria, acreditamos se tratar de um paleocanal. Para confirmar as interpretações discutidas neste texto, serão necessárias novas etapas de campo para um levantamento de GPR 3D e sondagens com vibracore.

\section{Discussão e Conclusões}

O comportamento morfodinâmico da região da foz deste estuário indica que a maior parte do sedimento depositado neste local é oriunda de setores localizados a leste do estuário. Este sedimento é resultado do retrabalhamento de sedimento praial, transportado pela deriva litorânea (Aquino da Silva et al., 2010). A evolução dos spits, bem como a morfodinâmica dos bancos arenosos dentro canal fluvial, mostram que as correntes de maré enchente predominam no que diz respeito ao transporte sedimentar. É provável que o spit leste não suporte as condições hidrodinâmicas geradas pela evolução deste senário em que ocorre a progradação do spit leste (para NO) e estreitamento da foz do estuário (Aquino da Silva et al., 2011). Com isto, é provável que ocorra o seccionamento do spit leste, fazendo com que a configuração morfológica da área se assemelhe ao encontrado em 2010. Isto mostra que há uma ciclicidade no processo evolutivo ocorrido na foz deste estuário (Aquino da Silva et al., 2011).

Adicionalmente, por meio do dado de GPR foram observados padrões de reflexões associados ao crescimento de um dos spits. Dentre eles, destacam-se um conjunto de refletores com mergulhos de SO para $\mathrm{NO}$, em profundidade de até $3 \mathrm{~m}$, interpretados como um registro pretérito do crescimento do spit. Adicionalmente, foi identificado o contato entre sedimentos élicos e o topo do spit, além de um paleocanal. Finalmente, por meio da seção de GPR, foi verificada a presença de uma zona cega, típica de áreas com intrusão salina.

Uma interpretação conjunta de todos os resultados não permitiu definir claramente a dinâmica costeira local, sendo necessária a realização de novos estudos geológicos, geofísicos, oceanográficos e climatológicos.

\section{Agradecimentos}

Os autores agradecem ao Departamento de Geofísica da Universidade Federal do Rio Grande do Norte pelo fornecimento do GPR utilizado neste trabalho e também ao Departamento de Geologia pelo empréstimo do GPS RTK.

\section{Referências}

Aquino da Silva, A. G. A., Vital, H. and Silveira, I. M. 2011. Barrier Island Evolution. In P. and R. T. M. Rosati, J. D., Wang (Ed.), The Proceedings of The Coastal Sediments 2011 (p. 3000). World Scientific Publishing Co. Pte. Ltd.

Aquino da Silva, G. A., Vital, H., 2010. Estimation of Presidio's Barrier Island (Guameré-NE/Brazil) Migration by Means of GIS and Remote Sensing. In Rostock (Ed.), Coastline Reports (pp. 45-48). Die Küsten Union Deutschland e.V.

Conti, I.M.M., Oliveira Jr, J.G. \& Caldas, L.H.O. 2015. Avanço das dunas sobre residências na Praia de Barra 
do Rio - RN: O uso de GPR para visualização da arquitetura deposicional dos depósitos eólicos e praiais. 14th International Congress of the Brazilian Geophysical Society held in Rio de Janeiro, Brazil, August 3-6, 2015.

COSERN, 2003. Potencial Eólico do Estado do Rio Grande do Norte (www.cresesb.cepel.br/publicacoes/download/atlas eolic o/atlas eolico RN.pdf).

ESRI. 2012. ArcGIS Desktop: Release 10. Redlands, CA: Environmental Systems Research Institute.

GOOGLE, 2001. Google Earth. Version 7.1.5.1557. Barra do Rio. Disponível em: www.google.com.br/intl/ptBR/earth/. Acesso em: 13/04/2016.

GOOGLE, 2005. Google Earth. Version 7.1.5.1557. Barra do Rio. Disponível em: www.google.com.br/intl/ptBR/earth/. Acesso em: 13/04/2016.

GOOGLE, 2010. Google Earth. Version 7.1.5.1557. Barra do Rio. Disponível em: www.google.com.br/int//ptBR/earth/. Acesso em: 13/04/2016.

GOOGLE, 2015. Google Earth. Version 7.1.5.1557. Barra do Rio. Disponível em: www.google.com.br/int//ptBR/earth/. Acesso em: 13/04/2016.

Lima, J.S.D., 2011. Análise e Monitoramento Geoambiental na Praia de Genipabu, Extremoz/RN. Dissertação de Mestrado, Programa de Pós-Graduação em Geografia/UFRN, Natal. 114p.

Lima, Z.M.C., VITAL H. 2002. Caracterização da Dinâmica Ambiental da Região Costeira do Municício de Galinhos, Litoral Norte do RN. II Workshop de Avaliação Annual dos PRH's-ANP da UFRN.

Oliveira. C., 1993. Curso de cartografia moderna. 2 ed. Rio de Janeiro: IBGE.

Sandmeier, K.J., 2000. REFLEXW Version 6.0, Windows $9 \times / 2000 /$ NT. Program for the processing of seismic, acoustic or eletromagnetic reflection, refraction and transmission data. Germany. 272 p.

Vilas, F., Arche A., Ferrero, M. \& Isla, F., 1999. Subantarctic macrotidal flats, cheniers and beaches in San Sebastian Bay, Tierra del Fuego, Argentina. Marine Geology, 160, $301-326$.

Xavier Neto, P. 2006. Processamento e Interpretação de Dados 2D e 3D de GPR: Aplicações no Imageamento de Feições Kársticas e Estruturas de dissolução no Campo de Petróleo de Fazenda Belém - CE. Tese de Doutorado em Geofísica Programa de Pós-Graduação em Geodinâmica e Geofísica/UFRN, Natal. 192 p. 


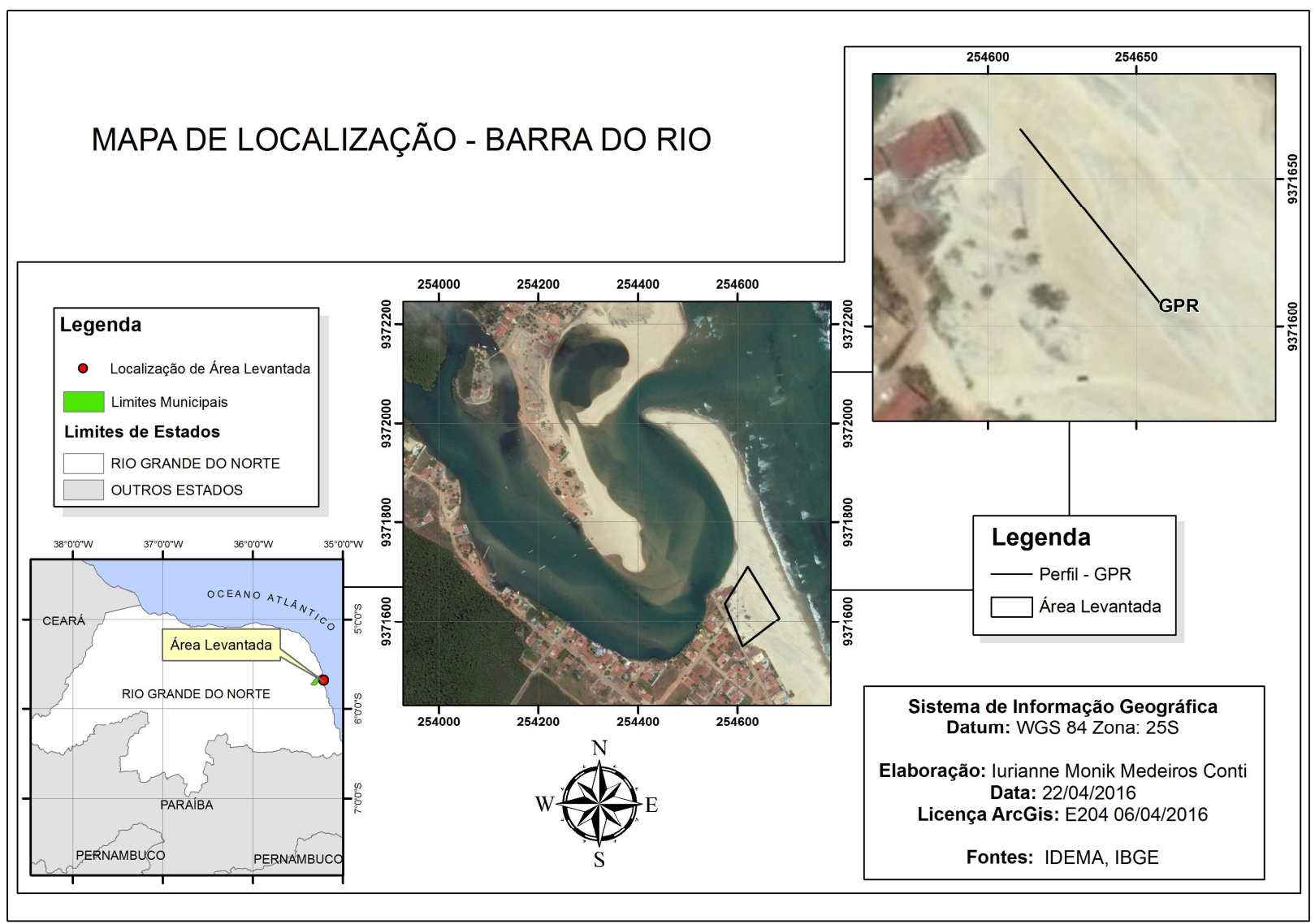

Figura 1 - Área de trabalho as margens do Rio Ceará Mirim, no município de Extremoz/RN. Por meio desta figura pode-se observar a posição da seção de GPR. 

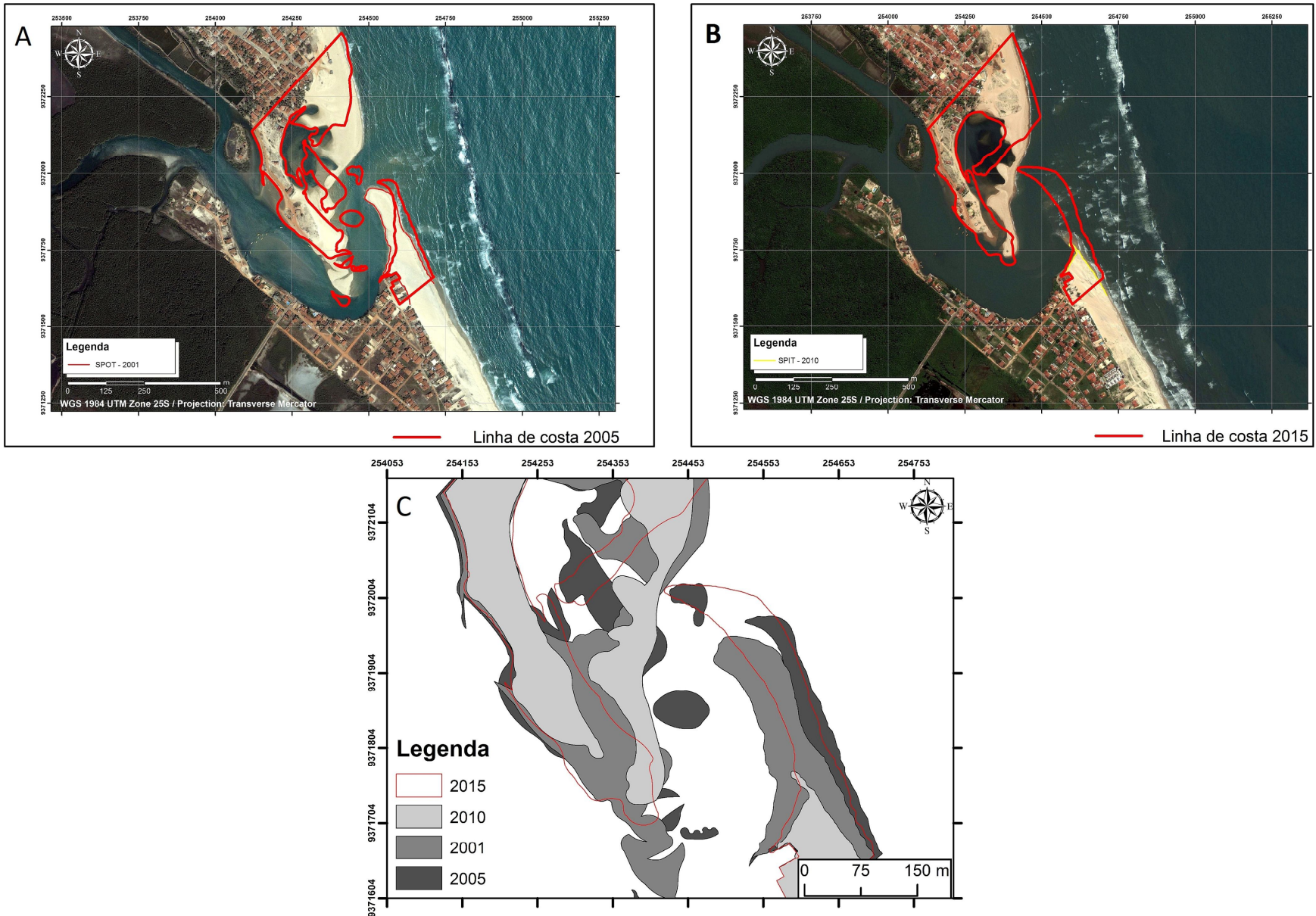

Figura 2 - A) Imagem de satélite obtida em 2001. Linha vermelha grossa indica a posição dos spits em 2010; B) Imagem de satélite obtida em 2010. Linha vermelha indica a posição do spit em 2015; C) Interpretação das relações de sedimentação/erosão observadas nos anos de 2001, 2005, 2010 e 2015. 

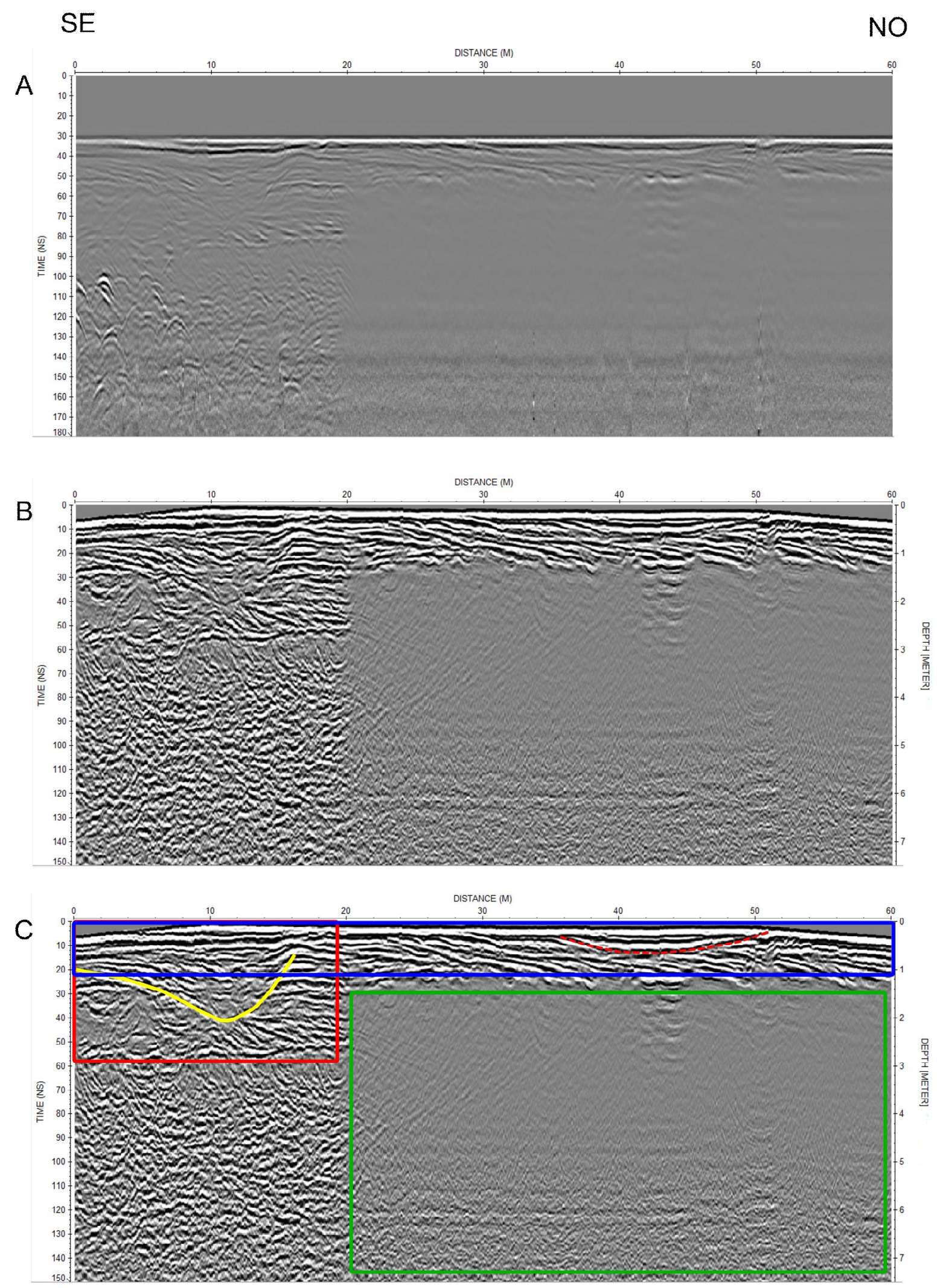

Figura 3 - Seção de GPR, obtida com antena de 200MHz. A)Dado bruto; B) Dado processado; C) Interpretação de 3B: Os retângulos vermelho/azul, verde $e$ as marcas amarela e vermelho pontilhado indicam, respectivamente: Refletores subhorizontais associados ao crescimento do spit, zona zaturada com água salgada, paleocanal e o contato entre sedimentos eólicos e praiais. 